\title{
35. JUNIPERUS PHOENICEA SUBSP. TURBINATA EN LA SIERRA DE GRAZALEMA
}

\author{
Montserrat ARISTA y Pedro L. ORTIZ
}

Palabras clave. Juniperus phoenicea, Cupressaceae, distribución.

Key words. Juniperus phoenicea, Cupressaceae, distribution.

Juniperus phoenicea L. presenta dos subespecies en la Península Ibérica: J. phoenicea subsp. phoenicea y J. phoenicea subsp. turbinata (Guss.) Nyman. Tradicionalmente se han distinguido las dos subespecies por su área de distribución (montañas del interior o arenales costeros respectivamente), por la morfología del gálbulo (globoso u ovoideo respectivamente) y por su tamaño (mayores en la subsp. turbinata), por el ápice de las ramas (netamente caudado en la subsp. turbinata y escasamente caudado en la phoenicea) (Amaral Franco, 1986; Fernández Galiano, 1987), o por caracteres fitoquímicos, sin prodelfinidina en la subsp. phoenicea y con prodelfinidina en la subsp. turbinata (Lebreton \& Thivend, 1981; Lebreton, 1983; Lebreton \& Rivera, 1988).

A pesar de que el área de distribución considerada para la subespecie turbinata son los arenales costeros, esta subespecie ha sido encontrada en zonas montañosas del interior, concretamente en la Sierra de Grazalema (Camilla alta, Arista y Ortiz, 11.11.1993, SEV 136065, 136065, 136066, 136067, 136068, 136069, 136070, 136071, 136072, 136073, 136074, 136075; Arista y Ortiz, 25.11.1994, SEV 136076, 136077, 136078, 136079, 136080, 136081, 136082, 136083, 136084, 136085, 136086) junto con la subespecie phoenicea (Arista y Ortiz, Puerto del Norno de la Miera, Sierra de Zafalgar, 12.3.1994, SEV 136087; 21.1.1995, SEV 136088; 10.2.1994, SEV 136092, 136093, 136094, 136095, 136097; 14.5.1994, SEV 136096, 136098).
En la Sierra de Grazalema, ambas subespecies se encuentran muy próximas entre sí, aunque sobre sustratos diferentes; la subespecie phoenicea se distribuye por suelos dolomíticos de la sierra de Zafalgar, mientras que la subespecie turbinata se encuentra sobre calizas cámbricas en las faldas del Bocaleones. Los frutos de ambas subespecies difieren significativamente en su tamaño: los de la subespecie turbinata son más grandes con una longitud media de $7,9 \pm 0,1 \mathrm{~mm}$ y un diámetro de $8,8 \pm 0,11 \mathrm{~mm}(\mathrm{n}=71)$, mientras que los de la subespecie phoenicea son de $6 \pm 0,1 \mathrm{~mm}$ de longitud y de $5,8 \pm 0,1 \mathrm{~mm}$ de diámetro $(\mathrm{n}=$ 142), $\mathrm{F}=114,17,1 \mathrm{gl}, \mathrm{p}<0,001$ para la longitud, y para el diámetro $\mathrm{F}=303,003,1 \mathrm{gl}, \mathrm{p}<0,001)$. Además de las diferencias morfológicas del fruto y de las ramas, ambas subespecies tienen separados sus momentos de floración en la Sierra de Grazalema. Juniperus phoenicea subsp. turbinata florece siempre en otoño, entre los meses de Octubre y Noviembre, mientras que la población de J. phoenicea subsp. phoenicea lo hace al final del invierno, en los meses de Febrero y Marzo.

Además de esta localidad, Juniperus phoenicea subsp. turbinata se encuentra en otras zonas montañosas de la Sierra de Líjar (Cádiz: Algodonales, Sierra de Líjar, calizas cámbricas, Aparicio, 26.4.1980, SEV 57419; Aparicio, 8.3.1980, SEV 57420) y de la Sierra de Benahavis (Málaga: Benahavis, ladera del río Guadalmina, Arista, Machado, Ortiz y Talavera, 2.2.1995, SEV 136043, 136144, 136145). 


\section{BIBLIOGRAFÍA}

AMARAL FRANCO, J. -1986- Juniperus. In Castroviejo, S., M. Laínz, G. López González, P. Montserrat, F. Muñoz Garmendia, L. Paiva y L. Villar (eds.). Flora Iberica, Vol. I Real Jard. Bot., Madrid, C.S.I.C

FERNANDEZ GALIANO, E. -1987- Juniperus. In Valdés, B., S. Talavera y E. Fernández Galiano (eds.). Flora Vascular de Andalucía Occidental. Ketres. Barcelona.

LEBRETON, Ph. -1983- Nouvelles données sur la distribution au Portugal et en Espagne des sousespèces du genévrier de Phénicie (Juniperus phoenicea L.). Agronomia Lusitanica, 42: 5662.
LEBRETON, Ph \& S. THIVEND -1981- Sur une sous-espèce du Genévrier de Phénicie Juniperus phoenicea L., définie à partir de critères biochimiques. Naturalia Monspeliensia, 47: 112.

LEBRETON, Ph \& RIVERA, D. -1988- Analyse du taxon Juniperus phoenicea L. sur des bases biochimiques et biométriques. Naturalia Monspeliensia, 53: 17-41.

Aceptado para su publicación en Junio de 1995

Dirección de los autores. Departamento de Biología Vegetal y Ecología. Universidad de Sevilla. Apdo. 1095. 41080 Sevilla.

\section{APORTACIONES A LA FLORA DE ANDALUCÍA: SIERRA MORENA (CÓRDOBA)}

Manuel MELENDO LUQUE, Eusebio CANO CARMONA y Francisco VALLE TENDERO

Palabras clave. Flora, corología, fitosociología, Córdoba, Andalucía.

Key words. Flora, corology, fitosociology, Córdoba, Andalusian.

Como resultado de un estudio llevado a cabo en el noreste de la provincia de Córdoba, aportamos datos florísticos que revisten diverso interés. Algunas de las citas constituyen una novedad para la provincia, en tanto que otras lo son para Andalucía occidental o Andalucía.

Amaranthus powellii S. Watson

CÓRDOBA: Cardeña, El Robledillo, 30SUH8637, $720 \mathrm{~m}$, 24-6-93, M. Melendo, GDAC 39348. Nitrófila, en cultivos de huerta. No conocemos ninguna cita anterior de esta especie para la provincia de Córdoba.

Myosotis sicula Guss.

CÓRDOBA: Venta del Charco, Alto de
Españares, 30SUH8827, 725 m, 30-4-92, M. Melendo, GDAC 39367. Borde de charcas poco profundas que no se desecan completamente durante el verano (Glycerio-Sparganion). Es la segunda cita para la provincia de Córdoba, la primera se debe a Devesa y Cabezudo (1978).

\section{Callitriche lusitanica Schotsman}

CÓRDOBA: Montoro, Arroyo Arenosillo, 30SUH7921, 280 m, 16-6-93, M. Melendo, GDAC 39369. Sumergida en arroyos de aguas someras que se desecan en verano; (comunidades de Callitricho-Batrachion). Este taxón ha sido citado con cierta frecuencia en el centro-occidente peninsular, pero existen muy pocas referencias para Andalucía (Jaén: 